\title{
Videolaryngoscopes: From A Vision Problem to A Communication Problem
}

\author{
Eugenio D. Martínez-Hurtado ${ }^{*}$, Míriam Sánchez-Merchante² and Manuel Ángel Gómez-Ríos ${ }^{3}$ \\ ${ }^{1}$ Consultant Anaesthetist at Infanta Leonor University Hospital, Department of Anesthesia and Intensive Care, Madrid, \\ Spain \\ ${ }^{2}$ Consultant Anaesthetist at Alcorcon Foundation University Hospital, Department of Anesthesia and Intensive Care, \\ Madrid, Spain
}

${ }^{3}$ Consultant Anesthesiologist at Complejo Hospitalario Universitario de A Coruña, Spain

Received: June 06, 2017; Accepted: August 20, 2017; Published: August 31, 2017

*Corresponding author: Eugenio D. Martínez-Hurtado, Consultant Anaesthetist at Infanta Leonor University Hospital, Department of Anesthesia and Intensive Care, Av. Gran Vía del Este, 80, Madrid, Spain, Tel.:0034620283465; Fax: 0034911918000; E-mail: eugeniodaniel.martinez@salud.madrid.org

Safe airway management is one of the fundamental responsibilities of the anesthesiologist, and maintaining the gas exchange of the patient is a priority, something that will be achieved according to the context, clinical situation and environment where it is, regardless of the device with this is realized [1].

Inability to manage properly the airway can trigger lesions secondary to hypoxia in a few minutes, and almost two-thirds of the problems related to airway management will occur during anesthesia induction, with the orotracheal intubation and the inability to maintain correct ventilation as the main cause of anesthesia morbidity and mortality [2,3].

It is, therefore, the duty of the anesthesiologist to develop skills to be prepared before a difficult airway anticipated or not, thus increasing the margin of safety in their daily practices [4].

Actually, the gold standard for orotracheal intubation continues to be the direct Macintosh laryngoscope [5]. Since its appearance in 1943, many attempts have been made to improve technique and equipment as the technology has advanced. Direct laryngoscopes require the alignment of the oro-pharyngeal-laryngeal axes to visualize the glottis, as is well known, providing a limited view of the larynx, with a visual field around $15^{\circ}$ and sometimes with a distant and tube-like image, with insufficient light, or with the endotracheal tube interfering with vision.

Since the first videolaryngoscope appeared in 1996 (US patent $5,827,178$ ) to date, devices such as Glidescope (2001), Airtraq (invention of Spanish physician Pedro Acha, Spain, 2005, US Patent 6,843,769) or others (Pentax AWS, C-MAC, Trueview, King Vision, etc.) have emerged, up to the modern TOTALTRACK VLM, a latest generation video laryngeal mask that allows continuous patient ventilation to achieve intubation (Figure 1).

Videolaryngoscopes provide insight into the entry of glottis independent of the line of sight (indirect laryngoscopy), especially those with angled blades. The fact that the image sensor is located in the distal part of the blade allows a panoramic view of the glottis, without the need to "align the axes", which makes the hyperextension of the head unnecessary,

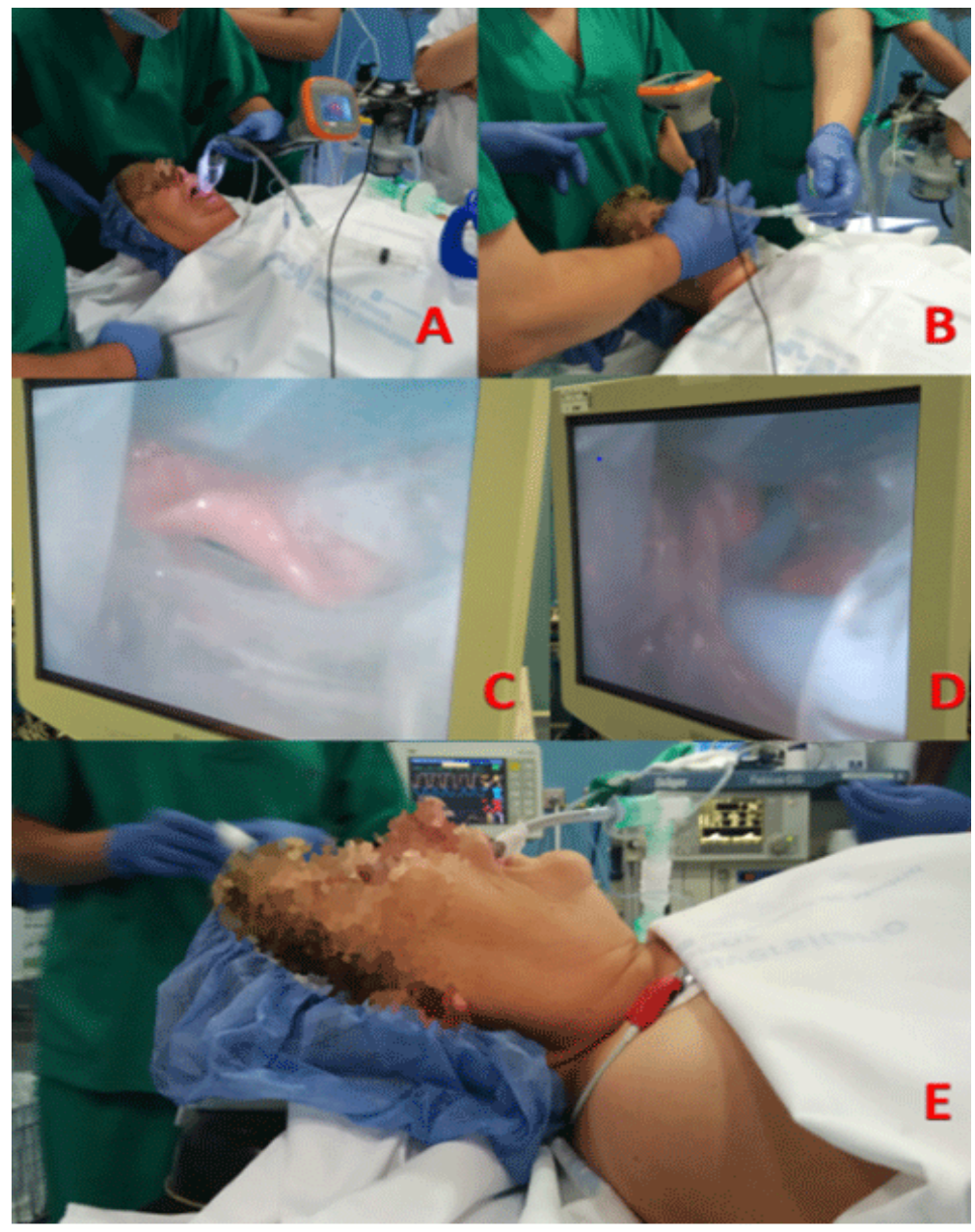

Figure 1: Intubation with Total track VLM of a patient with difficult airway predictors (A: introduction, B: evaluation while maintaining ventilation C: assessment of possible difficult intubation, D: use of Total track's Bougie introducer E: patient intubated successfully)

obtaining a laryngoscopy according to the Cormack-Lehane grade 1 or 2 scale (CL $1 / 4$ or $2 / 4$ ) in $99 \%$ of the cases [6].

It is impossible to know how many intubations are carried out daily using them, and clearly the main advantage of these devices depends not only on the "quantitative" improvement of laryngoscopy, but also on the quality in the image that allows recognizing anatomical structures, obtaining a laryngeal field of vision between $45^{\circ}$ and $60^{\circ}$ [7]. 
The image also provides certainty about both the success of intubation and the insertion depth of the endotracheal tube, allowing easy recognition and correction of esophageal intubation, as well as aiding the placement of a nasogastric tube under direct vision.

On the other hand, the LED light of the devices is of greater luminous intensity than the conventional one, possessing a spectral irradiation closer to the human eye [8]. In addition, indirect laryngoscopy facilitates teaching, since it allows simple learning of airway management techniques, facilitates continuous monitoring of the resident during intubation [9].

Currently, the international guidelines propose to use the videolaryngoscopes when the ventilation with face mask is effective and a previous attempt of intubation with a direct laryngoscope has been made. From this, it can be extrapolated that the percentage of videolaryngoscopes use would be equivalent to that of difficult intubation in non-urgent situations / scenarios, described around 5.8\% (95\% CI, 4.57.5\%) [10].

In the algorithm of the ASA of 2013 (11) is where videolaryngoscopes were included as rescue devices in case of unforeseen difficult airway and intubation failure, entering with force in daily practice. The British DAS has proposed to extend this recommendation and has announced that in the guidelines that will be issued by the end of 2015 videolaryngoscopes will be recommended as routine airway entry devices within plan a management.

However, the guides mention them in a generic way, without specifying or evaluating, which should be used, since there is no controlled work that reflects if one is superior to the rest, or not. The Difficult Airway Society (DAS) proposed the ADEPT study on "Evidence of Various Airway Devices," which would guide practitioners on the choice of a device, giving them the assurance of basing their decisions on scientific evidence and other anesthesiologist's experience [12].

In 2012, Healy et al. performed a systematic review with the aim of organizing the literature of efficacy of modern video laryngoscopes and then performing a quality assessment and making recommendations about use.

It was concluded that the use of Airtraq, CTrach (currently not marketed), GlideScope, Pentax-AWS and V-MAC was recommended in patients with risk of difficult laryngoscopy. In difficult direct laryngoscopy (CL> = 3) the use of Airtraq, Bonfils, Bullard, CTrach, GlideScope and Pentax-AWS was cautiously recommended by a practitioner with reasonable prior experience, and that there was evidence to support the use of Airtraq, Bonfils, CTrach, GlideScope, McGrath and Pentax-AWS after intubation failed by direct laryngoscopy [13].

Moreover, the 4th National Audit Project (NAP4) on major complications in airway management specifically mentioned the theoretical, but not proved, the benefit of video laryngoscopes, especially Airtraq, although the reported cases where they were used were scarce (12 de 184 cases [7\%]) and although these devices improved vision of the larynx, occasionally the endotracheal tube could not be successfully managed [14].

This difficulty in achieving intubation with a videolaryngoscope despite the correct exposure of the larynx, even in expert hands, often depends more on the practitioner's ability and the characteristics of the respiratory tract of the patient than the device used [15].

In 2015, the British DAS guidelines proposed the use of videolaryngoscopes as the first option because the choice of laryngoscope influences the chance of successful tracheal intubation, and videolaryngoscopes offer an improved view compared with conventional direct laryngoscopy. So, that all anesthetists should be trained to use, and have immediate access to, a videolaryngoscope [16].

Recently, new devices have appeared, a generation of videolaryngoscopes that allow the oxygenation of the patient, and the first model is the TOTALTRACK VLM. The fundamental idea is to ventilate and oxygenate the patient from the beginning at the end, regardless of the maneuver that is decided in the management of the airway, which allows the anesthesiologist to have more time to decide what strategy is the best for that particular airway according to the many particularities of the patient. Another of its main functions is to have a continuous airway vision system with an integrated anti-fog system. All this allows to improve the options of facing that airway management: it can be ventilated in laryngeal mask mode. It can be intubated in videolaryngoscope mode. It can be a self-rescue airway management by re-ventilating in laryngeal mask mode, or can be ventilated and intubated with a fiberoptic bronchoscope.

In this way, the TOTALTRACKVLM allows a global airway management strategy, with an initial approach while the patient is ventilated, an optimal intubation attempt is made and if this attempt fails, during the rescue of this attempt with the same device, since the TOTALTRACK VLM rescues itself functioning as a supraglottic device, covering all the plans proposed by the various international guides $[11,16]$.

However, as we know, the failure of endotracheal intubation through direct or indirect laryngoscopy usually occurs unexpectedly, despite the data of the predictors. And, despite the important morbidity and mortality associated with an Unpredictable Difficult Airway, later information on it is frequently deficient or inadequate, which complicates follow-up and approaches in subsequent surgeries, especially by distinct anesthesiologists and / or in different hospitals [16-18]. And although the patient is informed of the difficulty encountered in managing his airway, either verbally or in writing, up to half of the patients will forget or lose this information [19].

Among the systems that have traditionally been proposed for direct laryngoscopy information transmission is, first, the CormackLehane scale. This scale, proposed in 1984, describes 4 degrees of glottic exposure during direct laryngoscopy, with the patient in a maximal "sniffing" position, complete muscle relaxation, firm traction, and firm external laryngeal manipulations. Consequently, it is accepted that the difficulty for intubation can be suspected and even confirmed when direct laryngoscopy qualifies a grade 3 or 4 of this classification [20]. This scale was modified by Cook in 2000, increasing grade 3 in grade $3 \mathrm{~A}$ and $3 \mathrm{~B}$ or $3 \mathrm{E}$, depending on whether or not the epiglottis is removed from the posterior wall [21].

However, achieving a glottis vision according to the Cormack-Lehane grade 1 or 2 scale in indirect laryngoscopy is not always reflected in 
successful intubation. This is relatively frequent in video laryngoscopes that have the curved blade, such as Glidescope, Airtraq and Pentax AWS, especially during the learning stage [22]. Moreover, in the systematic review by Healy et al. [13], the vision of the glottis was a desirable outcome but was not considered a target for the recommendation.

In 1997 Adnet et al. [23] proposed and validated a quantitative and objective scale with several complexity factors in orotracheal intubation (Intubation Difficulty Scale [IDS]). To do this, they collected 7 factors that had been related in the literature with difficult intubation. The IDS value results from the sum of the score of these factors, and a value between 1 and 4 indicates slight difficulty, while figures greater than 5 indicate difficult intubation. These 7 factors take into account all moments of intubation, while the Cormack-Lehane classification only considers the time of laryngoscopic vision.

More recently the POGO (Percentage of Glottic Observed) scale has been proposed, in which the percentage of the glottis visible during laryngoscopy is simply reflected [24].

Problem lies in what happens when the approach is performed directly with a videolaryngoscope and we find a Difficult Non-Predicted Airway. The aforementioned scales are designed and validated for direct laryngoscopy, and the use of the Cormack-Lehane classification to compare with video laryngoscopy is questionable, although it is used in all studies since there is no other alternative scheme [13]. Therefore, these authors suggested that a system incorporating the difficulty encountered during the passage of the endotracheal tube should be employed, such as describing the difficulty (easy, difficult or failed), with a record of the glottic view obtained (as a "modified Cormack-Lehane"), and the name of the device used.

Different authors have already proposed to integrate those images and / or videos recorded during intubation with the video laryngoscope in the system of electronic medical history, along with other descriptors of the airway, for later anesthesiologists to see the data in the future [7].

The Spanish health system ensures the right to health protection and care for all citizens as a constitutional right, configured as a coordinated set of health services of the State Administration and the autonomous communities with public financing, which guarantee the universality and free of charge of health services at the time of use [25].

Through the "Online Health" Program, the Digital Clinical History of the National Health System has been promoted as a coordination and cohesion mechanism that offers safer health care for patients and with greater margins of efficiency than paper history. In the different autonomous communities have been implemented computer systems through which you can consult medical documents in electronic format stored in the Information Systems of the Hospitals that are part of the health services network.

The implementation of the Digital Clinical History of the National Health System (HCDSNS) began in 2006 with the aim of guaranteeing citizens and health professionals access to the most relevant medical documentation for the health care of each patient. The HCDSNS model, which has more than 45 million histories, has been developed in different autonomous communities in a heterogeneous way (Figure 2).

\section{HCDSNS: Cobertura de Población SNS}

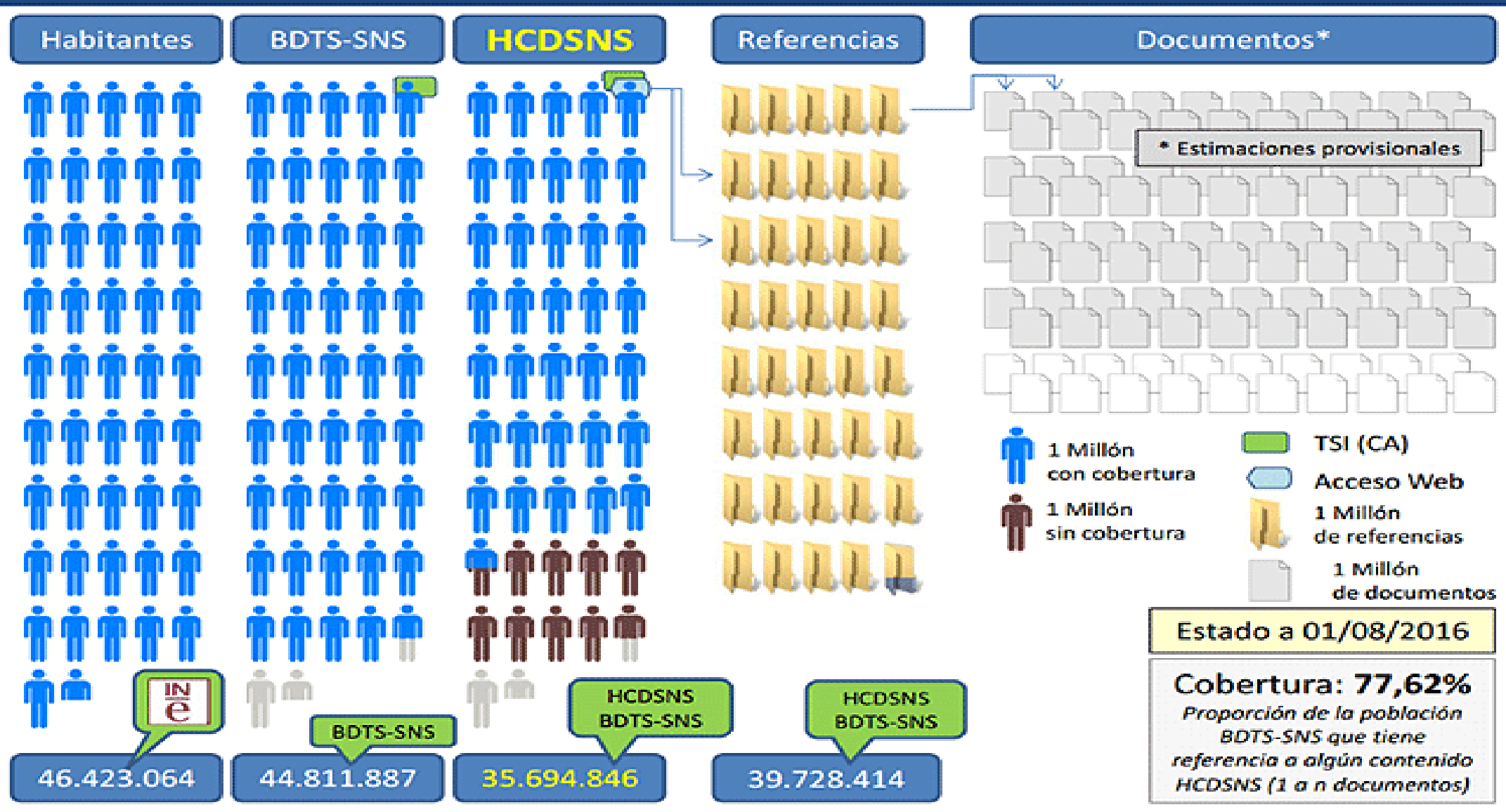

Figure 2: Digital Clinical History - population coverage in Spain - August 2016

Citation: Martinez-Hurtado E, Sanchez-Merchante M, et al. (2017) Videolaryngoscopes: From A Vision Problem to A Communication Problem. SOJ Anesthesio Pain Manag. 4(2):1-6. 
Only in the community of Madrid (SERMAS) there are more than 10 million image studies are accessible from any of the 30 hospitals (Figure 3 ). These clinical images are integrated into the Horus web application. Through an image viewer (WADO), we can access to any imaging test stored in the system through a service front architecture (Meta-WADO) Using a DICOM integration engine (HydraDICOM v.2.3 of Metaemotion) connects to retrieve and cache images of more than 28 PACS from different centers and providers (General Electric, Agfa, Siemens, Philips, Indra DCM4CHEE, etc.)

The objective in the next years is to make the images and videos obtained through videolaryngoscopy are introduced in a format of
"Videohistory" together with a description of the management of the airway of each patient. This would allow access to the data generated during the management of the patient's airway in previous interventions and would be included in the software through a form agreed by the Spanish Society of Anesthesia, Reanimation and Pain (SEDAR). It could be reflected the predictors observed during ventilation (HAN scale), vision obtained during intubation (Cormack-Lehane, pogo), the IDS scale, intubation information (tube used, attempts, preoxygenation, position, device, external maneuvers [Sellick, BURP], use of accessory material, such as a bougie, etc.), as well as images and / or videos obtained through videolaryngoscope.

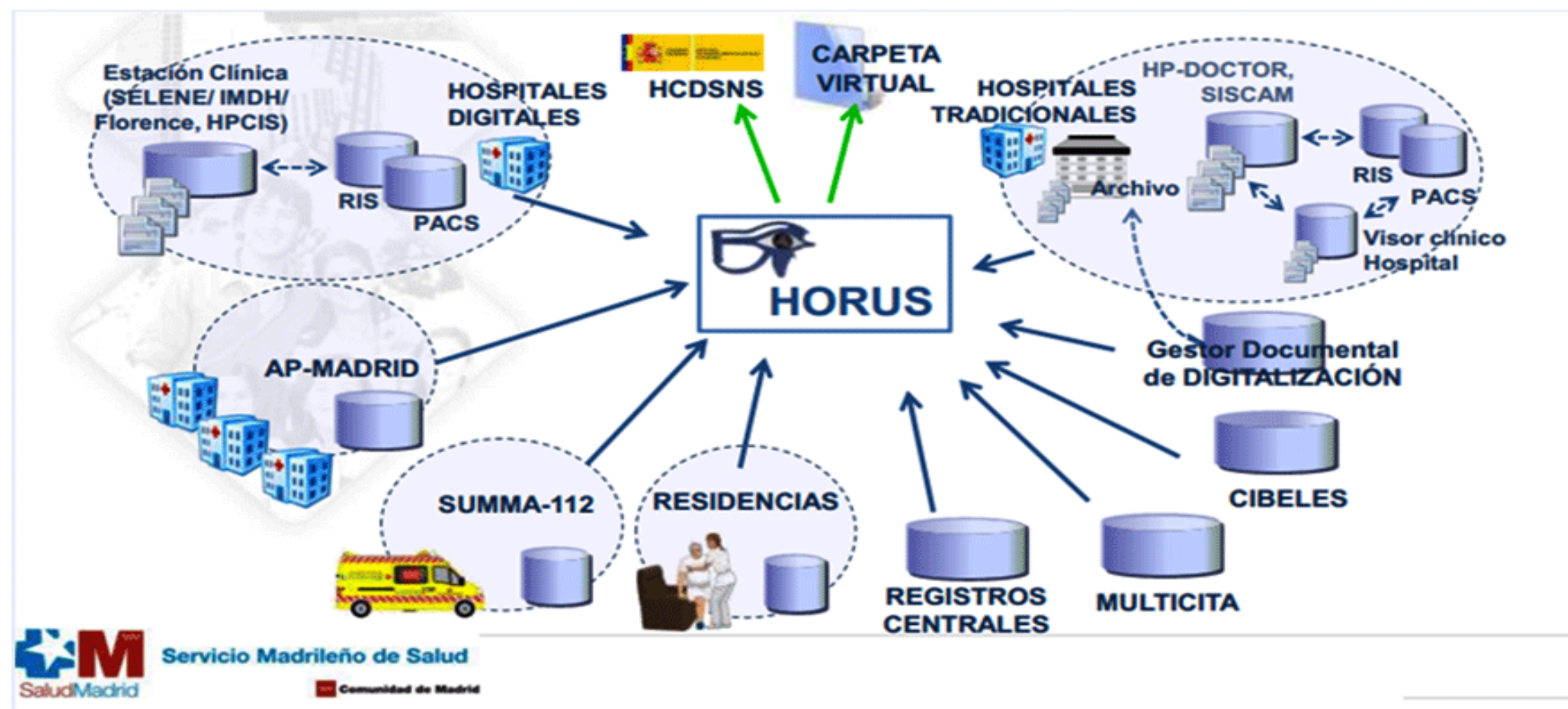

Figure 3: Horus data system

In these computer systems two objectives, health interoperability and allowing the patient to interconnect their clinical histories and dispose of them in any situation, allowing you to decide what you want to do with your data. Maintaining patient privacy must occupy a prominent place. Spain has legal norms, such as data protection law, biomedical research, patient rights, etc., which adequately regulate these aspects, although there is a need to improve how data management in the medical history will be managed, which define the profiles of the patients. Most health care organizations are developing similar information systems. In Europe, the project called epSOS is in the process of development and standardization. In the United States, where the health system is private, there is the Blue Button, a program that allows citizens to access their clinical information from the website of each health care provider, with the security procedure they determine.

This electronic clinical story will generate an enormous amount of information, data that will be analyzed through Big Data [26-28]. This mass health data management would allow the analysis of information from those millions of electronic medical records, including patient monitoring and telemedicine data, clinical order entry systems and medical prescriptions (electronic prescription, laboratory tests, shunts, etc.), decision supports systems, image storage and communication systems, and a long series of databases built for clinica, administrative or statistical purposes.

This is the so-called Real World Data (RWD), which allows us to analyze the benefits and adverse effects of health decisions in daily practice, unlike clinical trials and other studies conducted under controlled conditions, reflecting the patient's care in each specific context, and the medical outcomes they actually achieve.

The challenge for the analysis of great volumes of data is the development of huge, high-quality, multi-center databases. Investigating small datasets, Randomized Clinical Trial (RCT) results and large amounts of data can identify subgroups of patients who could benefit from a particular intervention, or it may be an alternative in the fields where performing an RCT is not possible.

The differences between "traditional" analytical and management applications and the new Big Data concepts are associated with 5 words, the "5 V": Volume, Variety and Velocity, Truth and Value of the data [28].

-The concept of Volume is very variable, and with each passing day, it raises what is considered "large volumes of data".

Citation: Martinez-Hurtado E, Sanchez-Merchante M, et al. (2017) Videolaryngoscopes: From A Vision Problem to A Communication Problem. SOJ Anesthesiol 
-Variety refers to the wide variety of sources of this data.

-Velocity refers to the speed with which data is received, processed and made decisions from it, because the data are out of date and lose their value quickly.

-The concept of Truthfulness reflects confidence in the data, and measures the usefulness of the data. Most of the incoming data are incomplete, with missing or incorrect fields, and reflects how quality data are extracted, eliminating the inherent unpredictability of some data, and thus achieving correct decision-making.

-Finally, the concept of Value refers to the importance of the data, which are the ones that should be analyzed and which should not.

Big Data analysis provides a layer of intelligence that goes beyond Evidence-Based Medicine (EBM), approaching personalized medicine that predicts and anticipates the health needs of patients and offers them the Most appropriate medical care.

Anesthetists will have access to large amounts of data from pre-, intra- and postoperative patient situation. The ability to analyze efficiently these data has important implications for the care and treatment provided, especially in the operating room, where the condition of a patient may change minute by the minute, and the ability to process data quickly facilitates the identification of pathological states and early treatment [29].

If we are able to detect risks in the health of patients, not only individually but also the population as a whole, we can use health resources in the most intelligent way and at a lower cost, something crucial in the moment we go through.

To date, the current global impact of videolaryngoscopes in the anesthetic literature has been hampered by the marked heterogeneity in the patient population. In addition to that generated by the wide variety of existing devices, the varying experience of practitioners, and the confusion generated when including mannequin studies. So we could, as Djulbegovic said, lose ourselves in the "fog of uncertainty", overwhelmed by the avalanche of data [30].

In a recent study conducted in 188,064 patients in Denmark that evaluated the airway preoperatively, conclusions about the ability of the usual or classic methods to detect the possible difficulty in their management were disappointing. And, perhaps, we should consider the need for a different approach [31].

However, although the prediction obtained with these isolated tests or multivariate models is poor, all the guides recommend preoperative assessment of airway $[11,14]$. And the Big Data analysis offers a powerful help system to discern between significant differences and those that are not [32].

Nevertheless, to infer the value to these data must be applied predictive models that allow us to optimize the care of patients. If we are able to detect risks in the health of patients, not only individually but also the population as a whole, we can use health resources in the most intelligent way and at a lower cost.

RWDs allow the development of sophisticated indicators to compare the quality of care received by patients cared for in different centers or by different physicians and to develop improvement strategies.

However, we must be cautious in substantiating the safety of our patients in clinical data analysis based on Big Data to detect the incidence of rare events, and be aware that most of the Difficult Airways are and will continue being Unforeseen.

Every failed intubation, emergency front-of-neck access, and airwayrelated unplanned admission should be reviewed and discussed, so that we must provide detailed clinical information with effective communication to other practitioners.

\section{References}

1. Hung O, Murphy M. Context-sensitive airway manegement. Anest Analg. 2010;110(4):982-983. doi: 10.1213/ANE.0b013e3181d48bbb

2. Peterson GN, Domino KB, Caplan RA, Posner KL, Lee LA, Cheney FW. Management of the Difficult Airway: A Closed Claims Analysis. Anesthesiology. 2005;103(1):33-39.

3. Mort TC. Emergency tracheal intubation: complications associated with repeated laryngoscopic attempsts. Anest Analg. 2004;99(2):607-613.

4. Valero y cols, S. Sabaté, R. Borràs, C. Áñez, S. Bermejo, F.J. González-Carrasco. Recomendaciones sobre seguridad del paciente quirúrgico. Protocolo de manejo de la VAD. Implicación de la Declaración de Helsinki. Revista Española de Anestesiología y Reanimación. Rev Esp Anestesiol Reanim. 2013;60(Supl 1):34-45.

5. Mulcaster JT, Mills J, Hung OR, MacQuarrie K, Law JA, PytkaS, et al. Laryngoscopic intubation: learning and performance. Anesthesiology. 2003;98(1):23-27.

6. Erol Cavus. Volker Dörges. The development of direct laryngoscopy. Trends in Anesthesia and Critical Care. 2014;4(1):3-9.

7. Zaouter C, Calderon J, Hemmerling TM. Videolaryngoscopy as a new standard of care. British Journal of Anaesthesia. 2015;114(2):181-183. doi: 10.1093/bja/aeu266

8. Lewis E, Zatman ST, Wilkes AR, Hall JE. Laryngoscope light output. Anaesthesia. 2009;64(6):688-689. doi: 10.1111/j.1365-2044.2009.05948.x

9. Ray DC, Billington C, Kearns PK, Kirkbride R, Mackintosh K, Reeve CS, et al. A comparison of McGrath and Macintosh laryngoscopes in novice users: a manikin study. Anaesthesia. 2009;64(11):1207-1210. doi: 10.1111/j.1365-2044.2009.06061.x

10.Shiga T, Wajima Z, Inoue T, Sakamoto A. Predicting difficult intubation in apparently normal patients: a meta-analysis of bedside screening test performance. Anesthesiology. 2005;103(2):429-437

11.American Society of Anesthesiologists Task Force on Management of the Difficult Airway. Practice guidelines for management of the difficult airway: an updated report by the American Society of Anesthesiologists Task Force on Management of the Difficult Airway. Anesthesiology. 2003;98:1269-1277.

12.Pandit JJ, Popat MT, Cook TM, Wilkes AR, Groom P, Cooke H, et al. The Difficult Airway Society 'ADEPT' guidance on selecting airway devices: the basis of a strategy for equipment evaluation. Anaesthesia. 2011;66(8):726-737. doi: 10.1111/j.13652044.2011.06787.x

13. Healy DW, Maties 0, Hovord D, Kheterpal S. A systematic review of the role of videolaryngoscopy in successful orotracheal intubation. BMC Anesthesiology. 2012;12:32. doi :10.1186 /1471-2253-12-32

14.Cook TM, Woodall N, Frerk C; Fourth National Audit Project. Major complications of airway management in the UK: results of the Fourth National Audit Project of the Royal College of Anaesthetists and the Difficult Airway Society. Part 1: anaesthesia. Br J Anaesth. 2011;106:617-631. doi: 10.1093/bja/aer058

15.Mosier JM, Whitmore SP, Bloom JW, Snyder LS, Graham LA, Carr GE, et al. Video laryngoscopy improves intubation success and reduces esophageal intubations compared to direct laryngoscopy in the medical intensive care unit. Crit Care. 2013;17(5):R237. doi: 10.1186/cc13061

16.Frerk C, Mitchell VS, McNarry AF, Mendonca C, Bhagrath R, Patel A, O’Sullivan EP; Difficult Airway Society intubation guidelines working group. Difficult Airway Society 2015 guidelines for management of unanticipated difficult intubation in adults. Br J Anaesth. 2015;115(6):827-848. doi: 10.1093/bja/aev371 
17.Hagberg C, Georgi R, Krier C. Complications of managing the airway. Best Pract Res Clin Anaesthesiol 2005;19:641-659.

18.Wilkes M, Beattie C, Gardner C, McNarry AF. Difficult airway communication between anaesthetists and general practitioners. Scott Med J. 2013;58:2-6. doi: $10.1177 / 0036933012474554$

19.Francon D, Bruder N. Why should we inform the patient safter difficult tracheal intubation? Ann Fr Anesth Reanim. 2008;27:426-30.

20.Cormack RS, Lehane J. Difficult tracheal intubation in obstetrics. Anaesthesia. 1984;39(11):1105-1111.

21.Cook TM. A new practical classification of laryngeal view. Anaesthesia. 2000;55(3):274279.

22.Larsson A, Dhonneur G. Videolaryngoscopy: towards a new standard method for tracheal intubation in the ICU? Intensive Care Med. 2013;39(12):2220-2222. do $10.1007 / \mathrm{s} 00134-013-3118-2$

23.Adnet F, Borron SW, Racine SX, Clemessy JL, Fournier JL, Plaisance P, et al. The intubation difficulty scale (IDS): proposal and evaluation of a new score characterizing the complexity of endotracheal intubation. Anesthesiology. 1997;87(6):1290-1297.

24.Ochroch EA, Hollander JE, Kush S, Shofer FS, Levitan RM. An outcome measure to assess glottic visualization: Validation of the percentage of glottic opening (POGO) scale [abstract]. Academic Emergency Medicine. 1999;6:506.
25.Ministerio de Sanidad, Servicios Sociales e Igualdad. Sistema Nacional de Salud. España. 2012. [monografía en Internet].

26.Levin MA, Wanderer JP, Ehrenfeld JM. Data, Big Data, and Metadata in Anesthesiology. Anesth Analg. 2015;121(6):1661-1667. doi: 10.1213/ANE.0000000000000716

27.Dutton RP. Large databases in anaesthesiology. Curr Opin Anaesthesiol. 2015;28(6):697702. doi: $10.1097 /$ AC0.0000000000000243

28.Raghupathi W, Raghupathi V. Big data analytics in healthcare: promise and potential. Heal Inf Sci Syst. 2014;2:3. doi: 10.1186/2047-2501-2-3

29.Giambrone GP, Hemmings HC, Sturm M, Fleischut PM. Information technology innovation: the power and perils of big data. Br J Anaesth. 2015;115(3):339-342. doi: $10.1093 / \mathrm{bja} / \mathrm{aev} 154$

30.Djulbegovic B. Lifting the fog of uncertainty from the practice of medicine. BMJ. 2004;329(7480):1419-1420.

31.Nørskov AK, Rosenstock CV, Wetterslev J, Astrup G, Afshari A, Lundstrøm LH. Diagnostic accuracy of anaesthesiologists' prediction of difficult airway management in daily clinical practice: a cohort study of 188064 patients registered in the Danish Anaesthesia Database. Anaesthesia. 2015;70(3):272-281. doi: 10.1111/anae.12955

32.Mathis MR, Haydar B, Taylor EL, Morris M, Malviya SV, Christensen RE. Failure of the Laryngeal Mask Airway Unique ${ }^{\mathrm{TM}}$ and $\mathrm{Classic}^{\mathrm{TM}}$ in the pediatric surgical patient: A study of clinical predictors and outcomes. Anesthesiology 2013;119:1284-1295. doi: 10.1097/ALN.0000000000000015 